\title{
ON SEMINORMAL INTEGRAL DOMAINS WITH TREED OVERRINGS
}

\author{
David E. Dobbs
}

Received: 26 August 2013

Communicated by Sait Halıcıŏlu

Dedicated to the memory of Professor Efraim P. Armendariz

\begin{abstract}
The following result uses and generalizes a recent result of Ayache on integrally closed domains. Let $R$ be a commutative integral domain with integral closure $R^{\prime}$ (inside the quotient field $K$ of $R$ ) such that each overring of $R$ (inside $K$ ) is a treed domain and there exists a finite maximal chain of rings going from $R$ to $R^{\prime}$. Then $R$ is a seminormal domain if and only if, for each maximal ideal $M$ of $R$, either $R_{M}$ is a pseudo-valuation domain or, for some positive integer $n$, there exists a finite maximal chain, of length $n$, of rings from $R_{M}$ to $\left(R_{M}\right)^{\prime}$ each step of which is (an integral minimal ring extension which is) either decomposed or inert. Examples are given in which the latter option holds where $R$ is one-dimensional and Noetherian.
\end{abstract}

Mathematics Subject Classification (2010): Primary 13B99, 13G05; Secondary 13F05, 13B21, 13E10

Keywords: Minimal ring extension, FCP, integral domain, overring, treed domain, pseudo-valuation domain, seminormal, integral closure, $i$-domain

\section{Introduction}

All rings considered below are commutative, with 1; and all inclusions of rings are (unital) ring extensions. As usual, the set of all prime (resp., maximal) ideals of a ring $R$ is denoted by $\operatorname{Spec}(R)$ (resp., $\operatorname{Max}(R)$ ). The "dim(ension)" of a ring $R$ will mean the Krull dimension of $R$. If $R$ is a (commutative integral) domain with quotient field $K$, then $R^{\prime}$ denotes the integral closure of $R$ (in $K$ ).

Let $R$ be a domain with quotient field $K$. By an overring of $R$, we mean any $R$-subalgebra of $K$, that is, any $\operatorname{ring} S$ such that $R \subseteq S \subseteq K$. As in [29], we say that $R$ is a pseudo-valuation domain if there is a valuation overring $V$ of $R$ such that $\operatorname{Spec}(R)=\operatorname{Spec}(V)$ as sets; it is enough to require that $R$ and the valuation overring $V$ have the same maximal ideal. When such a $V$ exists, it is uniquely determined by $R$ and is called the canonically associated valuation overring of $R$. As in [14] and [15], $R$ is called a locally pseudo-valuation domain (for short, an LPVD) if $R_{P}$ is a pseudo-valuation domain for each prime (equivalently, for each 
maximal) ideal $P$ of $R$. A pseudo-valuation domain is the same as a quasi-local LPVD. The most natural examples of LPVDs are Prüfer domains.

Let $R$ be a domain. As in [10], $R$ is said to be a divided domain if $P R_{P}=P$ for each $P \in \operatorname{Spec}(R)$; and a locally divided domain if $R_{P}$ is a divided domain for each prime (equivalently, for each maximal) ideal $P$ of $R$. A divided domain is the same as a quasi-local locally divided domain. The most natural examples of locally divided domains are Prüfer domains and one-dimensional domains. If $R$ is a locally divided domain, it is a going-down domain (in the sense of [8], [18]), but the converse is false [10, Proposition 2.1 and Example 2.9]. However, a partial converse does hold, as any seminormal going-down domain must be a locally divided domain (cf. [10, Corollary 2.6], [6, Corollary 3.5]). This explains in part our focus on seminormality as a tractable weakening of the "integrally closed" property in this work, as any LPVD is a seminormal domain (by [2, Lemma 2.1 (c) and Proposition 3.1 (a)]) and a locally divided domain [14, Corollary 2.3].

Recall that a domain $R$ is said to be a treed domain if $\operatorname{Spec}(R)$, as a poset under inclusion, forms a tree; that is, if no prime ideal of $R$ contains incomparable prime ideals of $R$. All the above-mentioned kinds of domains are examples of treed domains, since any going-down domain is a treed domain [8, Theorem 2.2]. Interest in domains $R$ all of whose overrings (including $R$ itself) are treed dates back to at least [12, Example 2.3], where the author constructed a domain $R$ all of whose overrings are treed such that $R$ is not a going-down domain. As that example was not integrally closed, we were led to ask if a quasi-local integrally closed domain $R$ must be a going-down domain if $R$ has valuative dimension 2 and is such that each overring of $R$ is treed (along with certain other conditions on $R$ that turned out to be extraneous). This question was answered in the affirmative by Ayache and Jarboui [4, Corollary 1]. Subsequently, the investigation was deepened by Ayache, Jarboui and Massaoud [5], in a study of pairs of domains all of whose intermediate rings are treed. Very recently, Ayache [3, Theorem 10] has proved that if an integrally closed domain $R$ is such that each overring of $R$ is a treed domain, then $R$ is an LPVD. This raises the following question, which returns to the spirit of [12]: what can be said along the lines of [3] if $R$ is not integrally closed (but is such that each of its overrings is treed)? Put differently, to what, if any, extent can such $R$ fail to be an LPVD? The main result of this note answers that question (see Corollary 2.6 ), by showing, under some reasonable assumptions that control the size of the extension $R \subseteq R^{\prime}$, that if $R$ is seminormal, the only alternative to $R$ being an LPVD involves finite chains of certain kinds of minimal ring extensions (in the sense of [23]), including one kind that was first considered by Gilmer and Heinzer [27, Example 4.3]. The domains in Example 2.7 illustrate the kind of complexity 
which is summarized in Corollary 2.6 but which cannot arise in [3]'s context of integrally closed domains.

Our basic reference on seminormality will be [36], although the original reference on the subject [38] has aged well. If $R \subseteq S$ are rings, we may say (thanks to [36, Theorem 2.5]) that $R \subseteq S$ is a seminormal extension (or that $R$ is seminormal in $S$ ) if $u \in S$ with $u^{2}, u^{3} \in R$ implies $u \in R$. While we will not need the definition of a "seminormal ring" here, it should be noted that the class of seminormal rings consists of certain reduced rings and that any integrally closed domain (in particular, any field) is a seminormal ring. It follows (cf. [36, Theorem 2.5 and Corollary 3.4]) that if $R$ is a domain with quotient field $K$, then $R$ is seminormal if and only if $R$ is seminormal in $K$. Seminormality makes its first appearance below in Proposition 2.2 , which gives a sufficient condition for pseudo-valuation domains. Remark 2.3 shows that Proposition 2.2 would fail without its seminormality hypothesis.

We mentioned above that our main results are made possible by "control(ling) the size of the extension $R \subseteq R^{\prime \prime \prime}$. More precisely, we assume in those results that $R \subseteq R^{\prime}$ satisfies the FCP property, in the sense that each chain (with respect to inclusion) of rings contained between $R$ and $R^{\prime}$ is finite. (Of course, it is reasonable to enlarge upon the domain-theoretic work in [3, Theorem 10] by requiring $R \subseteq R^{\prime}$ to satisfy FCP, as this is trivially the case when $R$ is an integrally closed domain.) While there has been much work on ring extensions satisfying the FCP property, especially for domains, during the past 15 years, everything that we will need about FCP can be found in our recent paper [21] with G. Picavet and M. PicavetL'Hermitte. Note that if a ring extension $R \subseteq S$ satisfies FCP, then there exist a non-negative integer $n$ and a finite maximal chain of rings $R=A_{0} \subset \ldots \subset A_{n}=S$; then, necessarily, $A_{i} \subset A_{i+1}$ is a minimal ring extension for all $i=0, \ldots, n$. The background on minimal ring extensions that is needed for this paper is summarized prior to Lemma 2.4.

Theorem 2.5 gives a characterization of the seminormal domains $R$ such that $R \subseteq R^{\prime}$ satisfies FCP. It should be noted that the proof of this result uses some facts from [21] about rings that may not be domains. (Thus, although our applications are to domains, we deviate from some of the literature on the FCP property where "ring" has tacitly meant "domain".) Theorem 2.5 leads directly to Corollary 2.6, where we give our extension of the motivating result of Ayache [3, Theorem 10]. Given the role of pseudo-valuation domains in [3, Theorem 10] and Corollary 2.6, it seems worthwhile to deepen the study of pseudo-valuation domains; this work does so through the inclusion of two results (Propositions 2.1 and 2.9) from the unpublished dissertation of M. S. Gilbert [26]. These two results are not needed for Theorem 2.5 or its applications, but as explained below, they do provide some motivation for the formulation of Corollary 2.6. 
As usual, $\subset$ denotes proper inclusion. Any unexplained material is standard, as in $[32]$.

\section{Results}

Recall from [34] that a domain $R$ is called an $i$-domain if the canonical contraction $\operatorname{map} \operatorname{Spec}(T) \rightarrow \operatorname{Spec}(R)$ is an injection for each overring $T$ of $R$. A domain $R$ is an $i$-domain if and only if $R_{M}$ is an $i$-domain for each $M \in \operatorname{Max}(R)$. If $R$ is a quasi-local domain, then $R$ is an $i$-domain if and only if $R^{\prime}$ is a valuation domain [34, Corollary 2.15]. Every overring of an $i$-domain is a going-down domain [34, Corollary 2.13] (cf. also [9, Corollary 2.5]).

We will begin with a result from the unpublished doctoral dissertation of M. S. Gilbert [26]. We next give that result and, for the sake of completeness, include Gilbert's proof.

Proposition 2.1. ( [26, Theorem 1.26]) Let $(R, M)$ be a quasi-local $i$-domain which is also a divided domain and is such that $R^{\prime}$ is comparable (with respect to inclusion) with each overring of $R$. Then:

(a) $\operatorname{Spec}(R) \backslash \operatorname{Max}(R)=\operatorname{Spec}\left(R^{\prime}\right) \backslash \operatorname{Max}\left(R^{\prime}\right)$.

(b) If, in addition, $R$ is not a field, then exactly one of the following two conditions holds:

(i) Both $R$ and $R^{\prime}$ have a (unique) largest nonmaximal prime ideal; or

(ii) In both $R$ and $R^{\prime}$, the maximal ideal is the union of the nonmaximal prime ideals.

(c) If the above condition (ii) holds, then $R$ is a pseudo-valuation domain.

Proof. (a) Let $P \in \operatorname{Spec}(R) \backslash \operatorname{Max}(R)$. Then $R_{P}$ is a proper overring of $R$ which is not integral over $R$ (cf. [32, Exercise 10, page 24]), and so the hypotheses give that $R^{\prime} \subset R_{P}$. Then $R_{P}$, being an overring of the valuation domain $R^{\prime}$, must be a valuation domain. Since $R$ is a divided domain, the maximal ideal of $R_{P}$ is $P R_{P}=P$. It follows that $P=P \cap R^{\prime}=P R_{P} \cap R^{\prime}$ is a prime ideal of $R^{\prime}$. Since $R$ is an $i$-domain and $R^{\prime}$ is an integral overring of $R$, there is a unique prime ideal of $R^{\prime}$ lying over any given prime ideal of $R$ (cf. [32, Theorem 44]). This argument shows that if $P \in \operatorname{Spec}(R) \backslash \operatorname{Max}(R)$, then $P$ is the unique prime ideal of $R^{\prime}$ that lies over $P$; and, by the going-up property of integral extensions (cf. [32, Theorem 44]), $P \notin \operatorname{Max}\left(R^{\prime}\right)$. By the incomparability property of integral extensions (cf. [32, Theorem 44]), each nonmaximal prime ideal of $R^{\prime}$ must contract to a nonmaximal prime ideal of $R$, and so the proof of (a) is complete.

(b) By the above comments, $R^{\prime}$ is a valuation domain (hence, a quasi-local treed domain); let $M^{\prime}$ denote the maximal ideal of $R^{\prime}$. As the nonmaximal prime ideals of $R^{\prime}$ (equivalently, of $R$ ) form a chain, their union is a prime ideal, say $Q$, of $R^{\prime}$ [32, Theorem 9]. By (a), $Q$ is a prime ideal of both $R$ and $R^{\prime}$. Clearly, at most one 
of (i) and (ii) holds. To show that one of (i) and (ii) holds, it is enough to show that if $Q=M$, then $Q=M^{\prime}$. Now if $Q=M$, then $M$ is a prime ideal of $R^{\prime}$ that contracts to $M$, whence $M=M^{\prime}$ and $Q=M^{\prime}$.

(c) Suppose that (ii) holds. Then $M=Q=M^{\prime}$. As $R$ and its valuation overring $R^{\prime}$ then have a common maximal ideal, $R$ is a pseudo-valuation domain by $[29$, Theorem 2.7].

We next give an application of Proposition 2.1. We will often need the result [11, Remark 4.8 (b)] that if $R$ is a pseudo-valuation domain which is also an $i$-domain (that is, which is such that $R^{\prime}$ is a valuation domain), then $R^{\prime}$ is the canonically associated valuation overring of $R$.

Proposition 2.2. Let $(R, M)$ be a quasi-local $i$-domain such that each overring of $R$ is comparable with $R^{\prime}$ and is a treed domain. Suppose also that $R$ is seminormal. Then $R$ is a pseudo-valuation domain.

Proof. Since the contraction map $\operatorname{Spec}\left(R^{\prime}\right) \rightarrow \operatorname{Spec}(R)$ is an injection, it follows from integrality (cf. [32, Theorem 44]) that $R^{\prime}$ inherits the "quasi-local" property from $R$. Let $N$ denote the maximal ideal of $R^{\prime}$. Next, since $R$ is an $i$-domain, it is a going-down domain. Hence, as $R$ is also seminormal and quasi-local, it must be a divided domain (as a consequence of [10, Corollary 2.6], as explained above).

Without loss of generality, $R$ is not a field. Suppose that we are in case (i) of Proposition 2.1 (b). Let $P$ denote the unique largest nonmaximal prime ideal of $R$ (resp., of $R^{\prime}$ ). As $R$ is a divided domain, $P R_{P}=P$. It is then easy to see (for instance, by using the " $u^{2}, u^{3}$ " criterion) that $R / P$ is a seminormal domain. Evidently, $\operatorname{dim}(R / P)=1$. Furthermore, as noted in [34, page 3], $R / P$ inherits the " $i$-domain" property from $R$. Therefore, by [2, Corollary 3.6], each overring of $R / P$ is an LPVD. In particular, $R / P$ is a pseudo-valuation domain. By the above remark, since $R / P$ is also an $i$-domain, it follows that its integral closure must be its canonically associated valuation overring, $V$.

Now, as in the proof of Proposition 2.1 (a), we get $R^{\prime} \subset R_{P}$. Therefore, applying $[24$, Corollary $1.5(5)]$ to the pullback $R_{P} \times_{R_{P} / P} R / P=R$, we get that $R^{\prime}=$ $R_{P} \times_{R_{P} / P} V$, and so $V=R^{\prime} / P$. Since $R / P$ and $V$ have the same maximal ideal, it follows that $M / P=N / P$, and so $M=N$. As $R^{\prime}$ is a valuation domain, $R$ must be a pseudo-valuation domain (cf. also [2, Lemma 3.4]).

By Proposition 2.1 (b), there is only one remaining case, namely, case (ii): in both $R$ and $R^{\prime}$, the maximal ideal is the union of the nonmaximal prime ideals. Hence, by Proposition 2.1 (a), the maximal ideals of $R$ and of $R^{\prime}$ are the same (since they are each the union of the same set of nonmaximal prime ideals). Thus, since $R^{\prime}$ is a valuation domain, $R$ must also be a pseudo-valuation domain in this case. 
Recall (cf. [23]) that a pair of distinct rings $R \subset S$ forms a minimal ring extension if there is no ring $T$ such that $R \subset T \subset S$. This usage should not be confused with [27]'s terminology of "unique minimal overring", which we shall avoid (other than to observe that if $R \subset S$ and $S$ is a/the "unique minimal overring" of $R$, in the sense of [27], then $R \subset S$ must be a minimal ring extension).

Remark 2.3. Proposition 2.2 would fail without the hypothesis that $R$ is seminormal. To see this, let $X$ be an analytic indeterminate over a field $K$, and put $R:=K+X^{2} K[[X]]$. (This construction belongs to a family of domains introduced by Gilmer and Huckaba in [28, page 429]. It was shown in [28, Proposition 11] that the overrings of $R$ are linearly ordered by inclusion, but we will give a direct proof of the part of this that we need.) Then $R^{\prime}=K[[X]]$ is a valuation domain, and so $R$ is a quasi-local $i$-domain. However, $R$ is not a pseudo-valuation domain. (Indeed, if $R$ were a pseudo-valuation domain, it would follow, as in the proof of Proposition 2.2, that its maximal ideal is the same as the maximal ideal of $R^{\prime}$, namely, $X K[[X]]$, but the maximal ideal of $R$ is $M:=X^{2} K[[X]]$.) Moreover, $R$ is a Noetherian ring by Eakin's Theorem [22, Theorem 2], since $R^{\prime}=R \cdot 1+R X$ is a finitely generated $R$-module; and $\operatorname{dim}(R)=\operatorname{dim}\left(R^{\prime}\right)=1$ by integrality (cf. [32, Theorem 48]). Hence, by the Krull-Akizuki Theorem [32, Theorem 93], each overring of $R$ is of dimension at most 1 and, therefore, must be a treed domain. To complete the proof that the "seminormal" hypothesis cannot be deleted from Proposition 2.2, it suffices to show that each overring of $R$ is comparable with $R^{\prime}$ and is treed. Note that since $R^{\prime}$ is a valuation domain, each overring of $R^{\prime}$ is a valuation domain and, hence, treed. Therefore, since $K[[X]]$ is a Prüfer domain, an application of [27, Theorem 2.4] shows that it suffices to prove that $R \subset R^{\prime}$ is a minimal ring extension. Since $R$ and $R^{\prime}$ have $M$ as a common ideal (in fact, $\left(R: R^{\prime}\right)=M$ ), it follows from [19, Lemma II.3] that it suffices to prove that $R / M \subset R^{\prime} / M$ is a minimal ring extension, that is, that $K \subset K \oplus K x$ is a minimal ring extension, where $x:=X+M \in K[[X]] / M$. As $x^{2}=0$, we see that $K \oplus K x \cong K[Y] /\left(Y^{2}\right)$, which is indeed a minimal ring extension of $K$ since its dimension as a vector space over $K$ is 2 (cf. also [23, Lemme 1.2]). This completes the proof.

The preceding two results show one kind of advantage in assuming that a domain is seminormal. Our main result, Corollary 2.6, will characterize the seminormal domains amongst the domains that do not stray overly far from their integral closures (in a sense that is made precise below) and have only treed overrings. Another noteworthy aspect is that Corollary 2.6 generalizes (but uses) the motivating result of Ayache [3, Theorem 10].

Prior to giving Corollary 2.6, we include an easy lemma for lack of a convenient reference. To prepare for that, we need to recall the following background material. It was shown in [23, Théorème 2.1 (i)] that each minimal ring extension $R \subset S$ 
has a crucial maximal ideal $M$, that is, a maximal ideal $M$ of $R$ such that the canonical injective ring homomorphism $R_{P} \rightarrow S_{P}$ is an isomorphism for all $P \in$ $\operatorname{Spec}(R) \backslash\{M\}$ while the analogous map $R_{M} \hookrightarrow S_{M}$ is a minimal ring extension. (Recall that if $D \subseteq E$ are rings and $P \in \operatorname{Spec}(D)$, then $E_{P}:=E_{D \backslash P}$.) In fact, the existence of such an ideal $M$ ensures that a ring extension $R \subseteq S$ is a minimal ring extension [13, page 14]. Our interest here is on minimal ring extensions that are integral. We next explain that there are three kinds of such extensions. Let $R \subset S$ be an integral minimal ring extension with crucial maximal ideal $M$. Then (cf. [19, Corollary II.2]) $M=(R: S)$; and, with $K:=R / M$, we say that the integral minimal ring extension $R \subset S$ is respectively decomposed, inert, or ramified according as to whether $S / M$ is isomorphic as a $K$-algebra to $K \times K$, a minimal field extension of $K$, or $K[Y] /\left(Y^{2}\right)$ for some indeterminate $Y$ over $K$. (These three cases correspond to the classification by Ferrand-Olivier [23, Lemme 1.2] of the minimal ring extensions of a field.) For $R \subset S$ and $M$ as above, $R_{M} \subset S_{M}$ is the same kind of integral minimal ring extension (that is, decomposed, inert or ramified) as $R \subset S$.

Lemma 2.4. If $R \subset S$ is an integral minimal ring extension which is either decomposed or inert, then $R$ is seminormal in $S$.

Proof. Let $M$ denote the crucial maximal ideal of $R \subset S$. By [36, Corollary 2.10], it is enough to prove that $R_{N}$ is seminormal in $S_{N}$ for each $N \in \operatorname{Max}(R)$. This is clear if $N \neq M$ (for then $R_{N}=S_{N}$ canonically). As the integral minimal ring extension $R_{M} \subset S_{M}$ would inherit the "decomposed" (resp., "inert") property from $R \subset S$, we can assume, without loss of generality, that $(R, M)$ is quasi-local. We will show that if $u \in S$ with $u^{2}, u^{3} \in R$, then $u \in R$. Note that if $u^{2} \notin M$, then $\left(u^{2}\right)^{-1} \in R$ and $u=u^{3}\left(u^{2}\right)^{-1} \in R$, as desired. Thus, without loss of generality, $u^{2} \in M$.

Suppose first that $R \subset S$ is decomposed. Then there are distinct maximal ideals $N_{1}$ and $N_{2}$ of $S$ such that $M=N_{1} \cap N_{2}$. It suffices to prove that $u \in N_{1} \cap N_{2}$. We fix $i \in\{1,2\}$ and proceed to show that $u \in N_{i}$. Put $v:=u+N_{i} \in S / N_{i}$. Then $v^{2}=u^{2}+N_{i}=0 \in\left(R+N_{i}\right) / N_{i} \subseteq S / N_{i}$, since $u^{2} \in M \subseteq N_{i}$. As $S / N_{i}$ is reduced, $v=0$; that is, $u \in N_{i}$, as required.

Next, suppose that $R \subset S$ is inert. Then $R / M \hookrightarrow S / M$ is a minimal field extension. Put $w:=u+M \in S / M$. Then $w^{2}=u^{2}+M=0 \in S / M$ since $u^{2} \in M$. Since $S / M$ is reduced, it follows that $w=0$; that is, $u \in M \subseteq R$.

For the sake of completeness, we note that if an integral minimal ring extension $R \subset S$ is ramified, then $R$ is definitely not seminormal in $S$.

We next characterize the seminormal domains $R$ such that $R \subseteq R^{\prime}$ satisfies FCP. 
Theorem 2.5. Let $R$ be a domain such that there exists a finite maximal chain of rings going from $R$ to $R^{\prime}$. Then the following two conditions are equivalent:

(1) $R$ is a seminormal domain;

(2) For each maximal ideal $M$ of $R$, either $(i) R_{M}$ is integrally closed or (ii) for some positive integer $n$, there exists a finite maximal chain of rings $D_{0}=R_{M} \subset$ $D_{1} \subset \ldots \subset D_{n}=\left(R_{M}\right)^{\prime}$ such that for each $i \in\{0, \ldots, n-1\}, D_{i} \subset D_{i+1}$ is (an integral minimal ring extension which is) either decomposed or inert.

Moreover, when the above condition (2) (ii) holds, then the positive integer $n$ and the finite maximal chain of rings $\left\{D_{0}, \ldots, D_{n}\right\}$ may be chosen so that for some integer $j, 0 \leq j \leq n$, we have that the integral minimal ring extension $D_{k} \subset D_{k+1}$ is decomposed whenever $0 \leq k \leq j-1$ and inert whenever $j \leq k \leq n-1$.

Proof. Suppose that $M \in \operatorname{Max}(R)$. We know that $\left(R_{M}\right)^{\prime}=\left(R^{\prime}\right)_{M}$ and localization converts minimal ring extensions to either equalities or minimal ring extensions $[23$, Lemme 1.3]. It is now clear that $R_{M}$ inherits the hypotheses on $R$. As we have already recalled that "seminormal ring" is a local property of domains [2, Lemma 2.1 (c)], we may assume, without loss of generality, that $(R, M)$ is quasi-local.

$(2) \Rightarrow(1)$ : Assume (2). Then, since any integrally closed domain is seminormal, we may assume, without loss of generality, that there exists a finite maximal chain of rings $D_{0}=R_{M}=R \subset D_{1} \subset \ldots \subset D_{n}=\left(R_{M}\right)^{\prime}=R^{\prime}$ such that for each $i \in$ $\{0, \ldots, n-1\}$, the integral minimal ring extension $D_{i} \subset D_{i+1}$ is either decomposed or inert. By Lemma 2.4, $D_{i}$ is seminormal in $D_{i+1}$ for all $i=0, \ldots, n-1$. So, by [36, Corollary 2.7] (or [38, Lemma 1.2], or an easy induction on $n$ ), $D_{0}$ is seminormal in $D_{n}$; that is, $R$ is seminormal in $R^{\prime}$. Since $R^{\prime}$ is a seminormal ring, it now follows from [36, Corollary 3.4] that $R$ is also a seminormal ring.

$(1) \Rightarrow(2)$ : Assume (1). Without loss of generality, $R$ is not integrally closed. Next, since $R \subset R^{\prime}$ is an integral extension, [21, Theorem 4.2 (a)] shows that the assumption that there exists a finite maximal chain of rings from $R$ to $R^{\prime}$ implies that $R \subset R^{\prime}$ satisfies FCP. Let $T$ denote the $t$-closure of $R$ in $R^{\prime}$ (in the sense of, for instance, [21, Proposition 4.5]). As $R \subseteq T \subseteq R^{\prime}$, both $R \subseteq T$ and $T \subseteq R^{\prime}$ inherit the FCP property from $R \subseteq R^{\prime}$. Therefore, there exist a positive integer $n$ and a finite maximal chain of rings $D_{0}=R=R_{M} \subset D_{1} \subset \ldots \subset D_{n}=R^{\prime}=\left(R_{M}\right)^{\prime}$ such that $T=D_{j}$ for some $j, 0 \leq j \leq n$. It suffices to prove that the integral minimal ring extension $D_{k} \subset D_{k+1}$ is decomposed whenever $0 \leq k \leq j-1$ and inert whenever $j \leq k \leq n-1$.

The following observations will be useful. Since $R$ is a seminormal domain, it is the seminormalization of $R$ in $R^{\prime}$ (cf. [36, Corollary 4.2]). Thus, $R$ is seminormal in $R^{\prime}$ and, a fortiori, seminormal in $T$. Also, $R \subseteq T$ is an infra-integral extension. (Recall that "infra-integral" means that $R /(Q \cap R) \hookrightarrow T / Q$ have the same quotient field for each $Q \in \operatorname{Spec}(T)$.) 
We will show first that $D_{k} \subset D_{k+1}$ is a seminormal extension for each $k$. (The argument in this paragraph is due to Gabriel Picavet and Martine L'Hermitte, and the author is grateful to them for their kind permission to present that argument here.) Since $R \subset R^{\prime}$ satisfies FCP, it follows from [21, Theorem 4.2 (a)] that $R^{\prime}$ is a finitely generated $R$-module and $R /\left(R: R^{\prime}\right)$ is an Artinian ring. One can make a similar conclusion if $R^{\prime}$ is replaced by any $R$-subalgebra of $R^{\prime}$. For each index $k$, it is easy to check that that $D_{k} \subset D_{k+1}$ is a seminormal extension if and only if $D_{k} /\left(R: R^{\prime}\right) \subset D_{k+1} /\left(R: R^{\prime}\right)$ is a seminormal extension. Thus, by passing from $\left\{D_{i}\right\}$ to $\left\{D_{i} /\left(R: R^{\prime}\right)\right\}$, we may assume (but only in this paragraph) that $R$ (while temporarily possibly no longer a domain) is an Artinian ring. Now, since $R \subset R^{\prime}$ is seminormal, [21, Lemma 4.8] (or [38, Lemma 1.3]) shows that if $S$ is any ring such that $R \subset S \subseteq R^{\prime}$, we have that $(R: S)$ is a radical ideal of $S$; being module-finite over an Artinian ring, any such $S$ is also an Artinian ring, and so $(R: S)$ is an intersection of finitely many maximal ideals of $S$. Fix an index $k$ and a ring $S$ such that $D_{k} \subset S \subseteq R^{\prime}$. We can write $(R: S)=N_{1} \cap \cdots \cap N_{m}$ for some finite nonempty subset $\left\{N_{1}, \ldots, N_{m}\right\}$ of $\operatorname{Max}(S)$. Since $(R: S) \subseteq\left(D_{k}: S\right)$, we have a canonical surjective $S$-algebra homomorphism $S /(R: S) \rightarrow S /\left(D_{k}: S\right)$. As the Chinese Remainder Theorem gives $S /(R: S) \cong \prod_{i=1}^{m} S / N_{i}$ (and we know the ideals of a finite direct product of fields), the First Isomorphism Theorem shows that $S /\left(D_{k}: S\right)$ is $S$-algebra isomorphic to the direct product of some of the $S / N_{i}$, say (after relabeling) $\prod_{i=1}^{r} S / N_{i}$. Then, equating annihilators of these isomorphic $S$-modules, we get $\left(D_{k}: S\right)=\cap_{i=1}^{r} N_{i}$, which is a radical ideal of $S$. Therefore, by [21, Lemma 4.8], $D_{k}$ is seminormal in $R^{\prime}$. Thus, a fortiori, $D_{k} \subset D_{k+1}$ is a seminormal extension.

To complete the proof that $(1) \Rightarrow(2)$, we need only show that $D_{k} \subset D_{k+1}$ cannot be ramified. Let $\mathcal{M}$ denote the crucial maximal ideal of this minimal ring extension. If $D_{k} \subset D_{k+1}$ were ramified, then the extension $F:=D_{k} / \mathcal{M} \subset D_{k+1} / \mathcal{M}$ would be both seminormal and ramified, necessarily with $D_{k+1} / \mathcal{M}=F[y]$ where $y \in F[y] \backslash F$ satisfies $0=y^{2}\left(=y^{3} \in F\right)$, which contradicts that $F \subset F[y]$ is seminormal.

It remains only to prove the "Moreover" assertion. As above, choose the chain $\left\{D_{i}\right\}$ so that $T=D_{j}$ for some index $j$. If $0 \leq k \leq j-1$, then the extension $D_{k} \subset D_{k+1}$ is seminormal (by the above argument) and clearly infra-integral, and hence decomposed (cf. [21, page 406]). On the other hand, if $k=j$, then $T=D_{k} \subset D_{k+1}$ inherits the " $t$-closed" property from $T \subset R^{\prime}$, and then $D_{k} \subset D_{k+1}$ must be inert, since each integral $t$-closed minimal ring extension is inert [21, page 406]. Finally, suppose that $j<k \leq n-1$. Then, since $T \subset R^{\prime}$ is a $t$-closed extension that satisfies FCP, it follows from [21, Lemma 5.6] that $D_{k} \subset D_{k+1}$ is inert. The proof is complete.

We now present our extension of [3, Theorem 10]. 
Corollary 2.6. Let $R$ be a domain such that each overring of $R$ is a treed domain and there exists a finite maximal chain of rings going from $R$ to $R^{\prime}$. Then the following two conditions are equivalent:

(1) $R$ is a seminormal domain;

(2) For each maximal ideal $M$ of $R$, either (i) $R_{M}$ is a pseudo-valuation domain or (ii) for some positive integer $n$, there exists a finite maximal chain of rings $D_{0}=R_{M} \subset D_{1} \subset \ldots \subset D_{n}=\left(R_{M}\right)^{\prime}$ such that for each $i \in\{0, \ldots, n-1\}$, $D_{i} \subset D_{i+1}$ is (an integral minimal ring extension which is) either decomposed or inert.

Moreover, when the above condition (2) (ii) holds, then the positive integer $n$ and the finite maximal chain of rings $\left\{D_{0}, \ldots, D_{n}\right\}$ may be chosen so that for some integer $j, 0 \leq j \leq n$, we have that the integral minimal ring extension $D_{k} \subset D_{k+1}$ is decomposed whenever $0 \leq k \leq j-1$ and inert whenever $j \leq k \leq n-1$.

Proof. We will adapt the proof, and use the statement, of Theorem 2.5. Suppose that $M \in \operatorname{Max}(R)$. Since each overring of $R_{M}$ is an overring of $R$, it is now clear that $R_{M}$ inherits each of the hypotheses on $R$. Hence, as above, we may assume, without loss of generality, that $(R, M)$ is quasi-local.

We recalled in the Introduction that any pseudo-valuation domain must be seminormal [2, Proposition 3.1 (a)]. Thus, by Theorem 2.5, it remains only to prove that if $R$ is integrally closed, then $R$ is a pseudo-valuation domain. Therefore, as $R$ is quasi-local (and all its overrings are assumed to be treed), an application of [3, Theorem 10] completes the proof.

We next give two examples of one-dimensional Noetherian seminormal domains $R$ that satisfy the second alternative (ii) in Corollary 2.6 (2). The ring $R$ in Example 2.7 (a) illustrates a paradigm suggested by [27, Corollary 2.4], inasmuch as $R \subset R^{\prime}$ is not a minimal ring extension but each localization at a maximal ideal of $R$ converts $R \subset R^{\prime}$ into either an identity or a minimal ring extension. On the other hand, the ring $R$ in Example 2.7 (b) shows the need for the alternative (ii) in Corollary 2.6 (2), as this $R$ is not an LPVD. (Of course, its integral closure is an LPVD, by the motivating result of Ayache [3, Theorem 10].) One also needs the alternative (i) in Corollary 2.6 (2), as there do exist integrally closed pseudo-valuation domains all of whose overrings are treed. (Indeed, for an integrally closed domain, the assertion that $(1) \Rightarrow(2)$ in Corollary 2.6 is precisely the result of Ayache [3, Theorem 10].) We note also that these rings illustrate the final conclusion of Corollary 2.6 (or of Theorem 2.5).

First, we review some notation that is useful in dealing with quadratic algebraic number fields. Let $d$ be a nonzero square-free integer. Define $\omega_{d}$ to be either $(1+\sqrt{d}) / 2$ or $\sqrt{d}$, according as to whether $d \equiv 1(\bmod 4)$ or $d \equiv 2,3(\bmod 4)$. It is well known that the ring of algebraic integers of $\mathbb{Q}(\sqrt{d})$ is $\mathbb{Z}\left[\omega_{d}\right]=\mathbb{Z}+\mathbb{Z} \omega_{d}$. 
Example 2.7. (a) $R:=\mathbb{Z}\left[6 \omega_{17}\right]$ is a one-dimensional Noetherian seminormal domain that satisfies the hypotheses of Corollary 2.6. Moreover, $E_{0}:=R \subset E_{1}:=$ $\mathbb{Z}\left[3 \omega_{17}\right] \subset E_{2}:=\mathbb{Z}\left[\omega_{17}\right]=R^{\prime}$ is a finite maximal chain of rings from $R$ to $R^{\prime}$, with $E_{0} \subset E_{1}$ decomposed and $E_{1} \subset E_{2}$ inert. However, for each maximal ideal $M$ of $R$, the extension $R_{M} \subseteq\left(E_{2}\right)_{R \backslash M}$ is either an identity or a minimal ring extension.

(b) $R:=\mathbb{Z}\left[2 \omega_{17}\right]$ is a one-dimensional Noetherian seminormal domain that satisfies the hypotheses of Corollary 2.6. Moreover, there exists a maximal ideal $M$ of $R$ such that $D_{0}:=R_{M} \subset D_{1}=\mathbb{Z}\left[\omega_{17}\right]_{R \backslash M}=\left(R_{M}\right)^{\prime}$ is a decomposed (integral minimal ring) extension and $R_{M}$ is not a pseudo-valuation domain.

Proof. (a) Note that $R^{\prime}=\mathbb{Z}\left[\omega_{17}\right]$ is one-dimensional, and hence so is $R$. Also, as in Remark 2.3, it follows from Eakin's Theorem that $R$ is Noetherian. Hence, by the Krull-Akizuki Theorem, each overring of $R$ is treed. Moreover, $R$ is seminormal by [16, Corollary 4.5] (with $n=m=6$ and $d=17$ ). Also, since each subring of a ring of the form $\mathbb{Z}\left[\omega_{d}\right]$ with quotient field $\mathbb{Q}[\sqrt{d}]$ must be of the form $\mathbb{Z}\left[n \omega_{d}\right]$ for some positive integer $n$, it is clear that $E_{0}:=R \subset E_{1}:=\mathbb{Z}\left[3 \omega_{17}\right] \subset \mathbb{Z}\left[\omega_{17}\right]$ is a finite maximal chain of rings from $R$ to $R^{\prime}$. The minimality of the ring extensions $E_{0} \subset E_{1}$ and $E_{1} \subset E_{2}$ can also be seen via [35, Theorem 5.2], which also shows that $E_{0} \subset E_{1}$ is decomposed (since 2 splits in $\mathbb{Q}(\sqrt{17})$ ). Similarly, we check that $E_{1} \subset E_{2}$ is inert. (The point here is that 3 is inert in $\mathbb{Q}(\sqrt{17})$. As 3 is an odd prime distinct from 17 , this is a matter of checking that the Legendre symbol $\left(\frac{17}{3}\right)=-1$. By the Law of Quadratic Reciprocity, as in [31, Theorem 1 (c), page 53], $\left(\frac{17}{3}\right)=\left(\frac{3}{17}\right)$, which explains in part how we were led to the choice of $D_{1}$ in building this example.)

Let $N$ denote the crucial maximal ideal of $E_{0} \subset E_{1}$, and let $M \in \operatorname{Max}(R)$. If $M \neq N$, then $R_{M}=\left(E_{1}\right)_{R \backslash M}$ and the extension $R_{M} \subseteq\left(E_{2}\right)_{R \backslash M}$ is just $\left(E_{1}\right)_{R \backslash M} \subseteq$ $\left(E_{2}\right)_{R \backslash M}$, which, by [23, Lemme 1.3], is either an identity or a minimal ring extension. Finally, suppose that $M=N$. It suffices to show that $\left(E_{1}\right)_{R \backslash N}=\left(E_{2}\right)_{R \backslash N}$. By the proof of [35, Theorem 5.2] (and [19, Corollary II.2]), $N=\left(R: E_{1}\right)=2 E_{1}$. Hence, $1 / 3 \in\left(E_{1}\right)_{R \backslash N}$, and it follows that $\left(E_{1}\right)_{R \backslash N}=\left(E_{2}\right)_{R \backslash N}$, as desired.

(b) Arguing as in (a), we see that $R$ is one-dimensional and Noetherian, with all its overrings being treed domains. Moreover, $R$ is seminormal by [16, Corollary 4.5] (with $n=m=2$ and $d=17$ ); cf. also [33, Example 1] and [37, Theorem 4.4]. Also as above, we see that $A_{0}:=R \subset A_{1}:=\mathbb{Z}\left[\omega_{17}\right]$ is a minimal ring extension; and one can use [35, Theorem 5.2] to check that $A_{0} \subset A_{1}$ is decomposed. Let $M$ denote the crucial maximal ideal of $A_{0} \subset A_{1}$. Then the minimal ring extension $\left(A_{0}\right)_{M}=R_{M}=D_{0} \subset\left(A_{1}\right)_{R \backslash M}=\left(R_{M}\right)^{\prime}=D_{1}$ is also decomposed. But [15, Example 4, especially page 71] shows that $R_{N}$ is not a pseudo-valuation domain, for some uniquely determined prime ideal $N$ of $R$ that lies over $2 \mathbb{Z}$. Necessarily, $N=M$, since the localization of $R$ at any maximal ideal other than the crucial 
maximal ideal $M$ coincides with a (quasi-local) localization of $\mathbb{Z}\left[\omega_{17}\right]$ (which must be a discrete rank one valuation domain and, hence, a pseudo-valuation domain).

Remark 2.8. Condition (2) in Corollary 2.6 cannot be used to characterize arbitrary seminormal domains, because a seminormal domain $R$ need not be such that $R \subseteq R^{\prime}$ satisfies FCP. We next give an example of what is perhaps the simplest kind of pseudo-valuation domain $R$ that illustrates this point. Let $k \subset F$ be an infinite-dimensional algebraic field extension and $V=F+M$ a valuation domain with maximal ideal $M \neq 0$. By [29, Example 2.1], $R:=k+M$ is a pseudo-valuation domain and, hence, seminormal. (For a more baroque proof that $R$ is seminormal, one could use [1, Proposition 2.1], as applied to the pullback $V \times_{F} k=R$, noting that $V$ and $k$ are seminormal and $F$ is reduced.) However, there is an infinite chain of fields between $k$ and $F$ and so, since $k \cong R / M$ and $F \cong V / M$ canonically, it follows from [20, Proposition 3.2] that $R \subset V\left(=R^{\prime}\right)$ does not satisfy FCP.

By Proposition 2.2, if $R$ is a quasi-local $i$-domain satisfying the hypotheses and condition (1) in Corollary 2.6, then $R$ must be a pseudo-valuation domain. On the other hand, Proposition 2.9 will show that if one wished to generalize [3, Theorem 10] to some quasi-local domains $D$ that are neither $i$-domains nor integrally closed, then it would be necessary to consider some $D$ that are not pseudo-valuation domains. To some extent, we feel that this result explains intuitively why condition (2) in Corollary 2.6 needed to bifurcate into a first condition (i), which had an LPVD favor, and another condition (ii), whose formulation was not apparent from the existing literature. In this vein, note that the quasi-local domain $R_{M}$ in Example 2.7 (b) is neither an $i$-domain nor a pseudo-valuation domain.

Proposition 2.9 is taken from the unpublished dissertation [26]. For the sake of completeness, we include a proof.

Proposition 2.9. ([26, Theorem 1.31]) Let $(R, M)$ be a pseudo-valuation domain. Then $R^{\prime}$ is comparable with each overring of $R$ if and only if either $R$ is integrally closed or $R$ is an i-domain.

Proof. Let $V$ be the canonically associated valuation overring of $R$. Suppose first that $R^{\prime}$ is comparable with each overring of $R$. Without loss of generality, $R \neq R^{\prime}$. Our task is to show that $R^{\prime}=V$. We have that $R \subset R^{\prime} \subseteq V$ and $M$ is the unique maximal ideal of each of these three rings. Put $k:=R / M, F:=R^{\prime} / M$ and $L:=V / M$. It suffices to prove that $F=L$. As $F \subseteq L$, we need only obtain a contradiction from the existence of an element $\alpha \in L \backslash F$. By hypothesis (and a standard homomorphism theorem), $F$ is comparable with each ring that is contained between $k$ and $L$. As $\alpha \notin F$, it follows that $k[\alpha] \nsubseteq F$, and so $F \subset k[\alpha]$. In addition (cf. [24, Corollary 1.5(5)]), $F$ is the integral (algebraic) closure of $k$ in $L$, and so $\alpha$ is transcendental over $k$. Then $k$ is algebraically closed in $k[\alpha]$ and, $a$ 
fortiori, algebraically closed in $F$, although $k \subset F$ and $F$ is algebraic over $k$, the desired contradiction.

For the converse, assume that either $R$ is integrally closed or $R$ is an $i$-domain. Our task is to prove that $R^{\prime}$ is comparable with each overring of $R$. As this is clear if $R=R^{\prime}$, we may assume that $R$ is an $i$-domain; that is, $R^{\prime}$ is a valuation domain. Then, by $\left[11\right.$, Remark 4.8 (b)], $R^{\prime}=V$. In any event, since $R$ is a pseudo-valuation domain, we see from the proof of [7, Theorem 3.1] that $V$ is comparable with each overring of $R$, and so the proof is complete.

Remark 2.10. (a) We cannot replace the hypothesis in Proposition 2.9 that $R$ is a pseudo-valuation domain with the hypothesis that $R$ is an LPVD. To see this, use the ring $R$ constructed in [17, Theorem 3.2], subject to the parenthetical comment in the statement of that result that we use data for the construction that satisfy $V_{i}=L+M_{i}$ and $W=k+M_{1}$ where $k \subset L$ is a minimal field extension. That construction is predicated on any integer $n>1$. As $R$ has exactly $n$ maximal ideals, $R$ is not a pseudo-valuation domain. However, $R$ is an LPVD, by parts (b) and (c) of $[17$, Theorem 3.2]. Moreover, $R$ is an $i$-domain because it is locally so (again by part (b) of [17, Theorem 3.2]). However, part (f) of [17, Theorem 3.2] produces exactly one overring of $R$ which is not comparable with $R^{\prime}$. In view of Example 2.7, it is interesting to note (via parts (e) and (d) of [17, Theorem 3.2]) that $R$ has the property that $\operatorname{dim}(R)=1$ and $R^{\prime}(\neq R)$ is a Prüfer domain.

(b) It is interesting to point out why the quasi-local $i$-domain $R:=K+X^{2} K[[X]]$ of Remark 2.3 cannot be used to illustrate Corollary 2.6. Recall that $R \subset R^{\prime}$ is a $n$ integral) minimal ring extension and that $R^{\prime}=K[[X]]$ is a Prüfer domain. Thus, by [27, Theorem 2.4], $R^{\prime}$ is comparable with each overring of $R$. Since $R$ is a onedimensional domain, it is now clear that each overring of $R$ is treed. However we have seen that $R$ is not a pseudo-valuation domain; so condition (2) (i) of Corollary 2.6 is not satisfied by $R$. Also, condition (2) (ii) of Corollary 2.6 is not satisfied by $R$, since $R \subset R^{\prime}$ is neither decomposed nor inert. Indeed, $R \subset R^{\prime}$ is ramified, since the proof of Remark 2.3 showed that $K:=R / M \subset R^{\prime} / M \cong K[Y] /\left(Y^{2}\right)$. Invoking either Proposition 2.2 or Corollary 2.6, we could conclude that $R$ is not a seminormal ring, but of course, the most natural way to get this conclusion would be to use the " $u^{2}, u^{3}$ " criterion.

(c) In closing, we point out why the quasi-local QQR-domain $(D, M)$ of $[27$, Example 4.3] cannot be used to illustrate Corollary 2.6. Note that $D$ is not a pseudo-valuation domain, by [29, Theorem 1.7], since its integral closure $D^{\prime}$ is not quasi-local. Thus, D does not satisfy condition (2) (i) in the statement of Corollary 2.6. However, $D$ does satisfy condition (2) (ii) in that statement, because it was essentially proved in [27] that $D \subset D^{\prime}$ is a decomposed (integral minimal ring) extension such that each proper overring of $D$ is a Prüfer (hence treed) domain. In 
addition, D satisfies condition (1) in the statement of Corollary 2.6; that is, $D$ is seminormal. One way to see that $D$ is seminormal is to combine Lemma 2.4 with [36, Corollary 3.4]; a second way is to let $k:=D / M$ and apply [1, Proposition 2.1] to the pullback $D^{\prime} \times_{k \times k} k=D$; and a third way is to use the following straightforward consequence of [25, Proposition 3.1] which was indicated to the author by Evan Houston [30]: each QQR-domain is seminormal. However, D cannot be used to illustrate Corollary 2.6 because $D$ fails to satisfy one of the riding hypotheses of that result. Indeed, D fails to be a treed domain. Moreover, [25, Remark 3.3] establishes, i.a., the following more general fact: if $A$ is a quasi-local $Q Q R$-domain and $A \subset A^{\prime}$ is (an integral minimal ring extension which is) decomposed, then $A$ is not a treed domain. In contrast, note that each of the base rings denoted by $R$ in [12, Examples 2.1 and 2.3] is a treed domain.

\section{References}

[1] D. F. Anderson and D. E. Dobbs, Fields in which seminormality implies normality, Houston J. Math., 16 (1990), 231-247.

[2] D. F. Anderson, D. E. Dobbs and J. A. Huckaba, On seminormal overrings, Comm. Algebra, 10 (1982), 1421-1448.

[3] A. Ayache, Integrally closed domains with treed overrings, Ricerche Mat., to appear.

[4] A. Ayache and N. Jarboui, An answer to a Dobbs conjecture about treed domains, J. Algebra, 320 (2008), 3720-3725.

[5] A. Ayache, N. Jarboui and E. Massaoud, Pairs of domains where all intermediate domains are treed, Arab J. Sci. Eng., 36 (2011), 933-946.

[6] A. Badawi and D. E. Dobbs, On locally divided rings and going-down rings, Comm. Algebra, 29 (2001), 2805-2825.

[7] E. Bastida and R. Gilmer, Overrings and divisorial ideals of rings of the form $D+M$, Michigan Math. J., 20 (1973), 79-95.

[8] D. E. Dobbs, On going down for simple overrings, II, Comm. Algebra, 1 (1974), 353-363.

[9] D. E. Dobbs, Ascent and descent of going-down rings for integral extensions, Bull. Austral. Math. Soc., 15 (1976), 253-264.

[10] D. E. Dobbs, Divided rings and going-down, Pac. J. Math., 67 (1976), 439458.

[11] D. E. Dobbs, Coherence, ascent of going-down and pseudo-valuation domains, Houston J. Math., 4 (1978), 551-567.

[12] D. E. Dobbs, On treed overrings and going-down domains, Rend. Mat., 7 (1987), 317-322. 
[13] D. E. Dobbs, Recent progress on minimal ring extensions and related concepts, in Commutative Rings: New Research, pp. 13-38, Nova Sci. Publ., Hauppage, New York, 2009.

[14] D. E. Dobbs and M. Fontana, Locally pseudo-valuation domains, Ann. Mat. Pura Appl., 134 (1983), 147-168.

[15] D. E. Dobbs and M. Fontana, On pseudo-valuation domains and their globalizations, in Proceedings of Trento Conference, Lecture Notes Math. 84, pp. 65-77, Dekker, New York, 1983.

[16] D. E. Dobbs and M. Fontana, Seminormal rings generated by algebraic integers, Mathematika, 34 (1987), 141-154.

[17] D. E. Dobbs and N. Jarboui, On integral domains with a unique overring that is incomparable with the integral closure, JP J. Algebra Number Theory Appl., 23 (2011), 1-24.

[18] D. E. Dobbs and I. J. Papick, On going down for simple overrings, III, Proc. Amer. Math. Soc., 54 (1976), 35-38.

[19] D. E. Dobbs, B. Mullins, G. Picavet and M. Picavet-L'Hermitte, On the FIP property for extensions of commutative rings, Comm. Algebra, 33 (2005), 121-134.

[20] D. E. Dobbs, G. Picavet and M. Picavet-L'Hermitte, On the maximal cardinality of chains of intermediate rings, Int. Electron. J. Algebra, 5 (2009), 391-429.

[21] D. E. Dobbs, G. Picavet and M. Picavet-L'Hermitte, Characterizing the ring extensions that satisfy FIP or FCP, J. Algebra, 371 (2012), 391-429.

[22] P. M. Eakin, Jr., The converse to a well known theorem on Noetherian rings, Math. Ann., 177 (1968), 278-282.

[23] D. Ferrand and J.-P. Olivier, Homomorphismes minimaux d'anneaux, J. Algebra, 16 (1970), 461-471.

[24] M. Fontana, Topologically defined classes of commutative rings, Ann. Mat. Pura Appl., 123 (1980), 331-355.

[25] M. Fontana and E. Houston, On integral domains whose overrings are Kaplansky ideal transforms, J. Pure Appl. Algebra, 163 (2001), 173-192.

[26] M. S. Gilbert, Extensions of commutative rings with linearly ordered intermediate rings, Ph. D. dissertation, University of Tennessee, Knoxville, 1996.

[27] R. Gilmer and W. J. Heinzer, Intersections of quotient rings of an integral domain, J. Math. Kyoto Univ., 7 (1967), 133-150.

[28] R. Gilmer and J. A. Huckaba, D-rings, J. Algebra, 28 (1974), 414-432.

[29] J. R. Hedstrom and E. G. Houston, Pseudo-valuation domains, Houston J. Math., 75 (1978), 137-147.

[30] E. G. Houston, personal communication, May 10, 2013. 
[31] K. Ireland and M. Rosen, A Classical Introduction to Modern Number Theory, second ed., Grad. Texts Math., 84, Springer, 1990.

[32] I. Kaplansky, Commutative Rings, rev. ed., Univ. Chicago Press, Chicago, 1974.

[33] A. Ooishi, On seminormal rings (general survey), Lecture Notes, RIMS, Kyoto Univ., 374 (1980), 1-17.

[34] I. J. Papick, Topologically defined classes of going-down domains, Trans. Amer. Math. Soc., 219 (1976), 1-37.

[35] M. Picavet-L'Hermitte, Seminormality and t-closedness of algebraic orders, in Advances in Commutative Ring Theory (Fez, 1997), pp. 521-540, Dekker, New York, 1999.

[36] R. G. Swan, On seminormality, J. Algebra, 47 (1980), 210-229.

[37] H. Tanimoto, Normality, seminormality and quasinormality of $\mathbb{Z}\left[m^{1 / n}\right]$, Hiroshima Math. J., 17 (1987), 29-40.

[38] C. Traverso, Seminormality and Picard group, Ann. Scuola Norm. Sup. Pisa, 24 (1970), 585-595.

\section{David E. Dobbs}

Department of Mathematics

University of Tennessee

Knoxville, TN 37996-1320, U.S.A.

e-mail: ddobbs1@utk.edu 